\title{
Spin and Orbital Magnetic Moment Anisotropies of Monodispersed Bis(Phthalocyaninato)Terbium on a Copper Surface
}

\author{
Sebastian Stepanow, ${ }^{*, \dagger}$ Jan Honolka, ${ }^{\dagger}$ Pietro Gambardella, ${ }^{\ddagger}$ Lucia Vitali, ${ }^{\dagger, \#}$ \\ Nasiba Abdurakhmanova, ${ }^{\dagger}$ Tzu-Chun Tseng, ${ }^{\dagger}$ Stephan Rauschenbach, ${ }^{\dagger}$ Steven L. Tait, ${ }^{\circ}$ \\ Violetta Sessi, ${ }^{\dagger}$ Svetlana Klyatskaya, ${ }^{\S}$ Mario Ruben, ${ }^{\S, l}$ and Klaus Kern ${ }^{\dagger, \perp}$ \\ Max-Planck-Institut für Festkörperforschung, Heisenbergstrasse 1, D-70569 Stuttgart, Germany, ICREA and Centre \\ d'Investigació en Nanociència i Nanotecnologia (ICN-CSIC), UAB Campus, E-08193 Barcelona, Spain, \\ IKERBASQUE, Basque Foundation for Science, E-48011, Bilbao, Spain, Centro de Fisica de Materiales \\ CFM-MPC, Centro Mixto CSIC-UPV, E-20018 San Sebastian, Spain, Department of Chemistry, Indiana University, \\ Bloomington, Indiana 47405, Institute of Nanotechnology, Karlsruhe Institute of Technology (KIT), \\ Hermann-von-Helmholtz-Platz 1, D-76344 Eggenstein-Leopoldshafen, Germany, IPCMS-CNRS UMR 7504, \\ Université de Strasbourg, 23 Rue du Loess, F-67034 Strasbourg, France, and Institut de Physique de la Matière \\ Condensée, Ecole Polytechnique Fédérale de Lausanne, CH-1015 Lausanne, Switzerland
}

Received June 11, 2010; E-mail: s.stepanow@fkf.mpg.de

\begin{abstract}
The magnetic properties of isolated $\mathrm{TbPc}_{2}$ molecules supported on a $\mathrm{Cu}(100)$ surface are investigated by $\mathrm{X}$-ray magnetic circular dichroism at $8 \mathrm{~K}$ in magnetic fields up to $5 \mathrm{~T}$. The crystal field and magnetic properties of single molecules are found to be robust upon adsorption on a metal substrate. The $\mathrm{Tb}$ magnetic moment has Ising-like magnetization; XMCD spectra combined with multiplet calculations show that the saturation orbital and spin magnetic moment values reach 3 and $6 \mu_{\mathrm{B}}$, respectively.
\end{abstract}

The assembly of molecular magnets on surfaces is a major challenge toward the engineering of molecule-based magnetoelectronic devices. One of the approaches explored to date is based on the self-assembly of molecular building blocks and transition-metal centers on metal substrates in ultrahigh-vacuum (UHV) conditions. This has led to the formation of two-dimensional surface-supported metal-organic networks with controllable magnetic anisotropy. ${ }^{1}$ Another promising route is the grafting of single-molecule magnets (SMMs) on surfaces, provided that the SMM magnetic properties measured in bulk samples survive the interaction with the substrate. Previous attempts have shown the difficulties in preparing SMM layers using standard surface deposition techniques. ${ }^{2}$ However, it was recently demonstrated that the magnetic character of compact $\mathrm{Fe}_{4} \mathrm{SMMs}_{4}$ in $\mathrm{x}$ situ prepared samples is preserved. ${ }^{3}$

Lanthanide bis-phthalocyaninato molecules represent a class of SMMs comprising a single rare-earth metal ion coordinated to two phthalocyanine $(\mathrm{Pc})$ molecules. ${ }^{4}$ In a combined scanning tunneling microscopy (STM) and density functional theory study it was shown that $\mathrm{TbPc}_{2}$ molecules can be transferred to a $\mathrm{Cu}(111)$ surface without damage using a printing technique, suggesting the survival of a large spin magnetic moment. ${ }^{5}$ Moreover, it was recently demonstrated that $\mathrm{TbPc}_{2}$ can be sublimated in UHV and that they self-assemble into highly ordered domains on a $\mathrm{Au}(111)$ surface. ${ }^{6}$ The question remains, however, if changes of the matrix environment around $\mathrm{TbPc}_{2}$ can appreciably alter the splitting of the crystal field levels and, hence, the magnetic behavior of the Tb ion. ${ }^{7}$

\footnotetext{
Max-Planck-Institut Stuttgart.

ICREA \& ICN Barcelona.

KIT Karlsruhe.

"Université Strasbourg.

${ }^{\perp}$ EPFL Lausanne.

Indiana University.

\# IKERBASQUE Bilbao \& CFM-MPC San Sebastian.
}

Here, we determine the magnetic properties of $\mathrm{TbPc}_{2}$ in contact with a metal surface using element-selective $\mathrm{X}$-ray magnetic circular dichroism (XMCD) measurements of isolated and oriented $\mathrm{TbPc}_{2}$ on a single-crystal $\mathrm{Cu}(100)$ substrate. XMCD spectroscopy allows us to separate the orbital and spin magnetic moments of the Terbium ion and probe their extraordinary anisotropic behavior. The experiments were carried out at the ID08 beamline of the European Synchrotron Radiation Facility. The samples were prepared in situ by thermal evaporation of $\mathrm{TbPc}_{2}$ onto the clean $\mathrm{Cu}(100)$ surface. The samples were first characterized by STM under UHV conditions and then transferred to the XMCD chamber without breaking vacuum. The integrity of the molecules after evaporation was proven by STM and mass spectrometry (see Supporting Information). X-ray absorption spectra (XAS) were acquired (total electron yield) for circular and linear light polarization in magnetic fields up to $5 \mathrm{~T}$ parallel to the X-ray direction. The sample temperature was varied between 300 and $8 \mathrm{~K}$. To compare the electronic and magnetic behavior of the molecules on a metal surface to their behavior in a bulk environment, we also investigated neutral, polycrystalline $\mathrm{TbPc}_{2}$ powders. Figure 1 shows an STM topograph of monodispersed $\mathrm{TbPc}_{2}$ molecules on the $\mathrm{Cu}(100)$ surface. STM shows that single $\mathrm{TbPc}_{2}$ units adsorb intact onto the substrate with the Pc plane parallel to the surface. The molecules do not aggregate due to the strong interaction of the lower Pc ligand with the substrate.

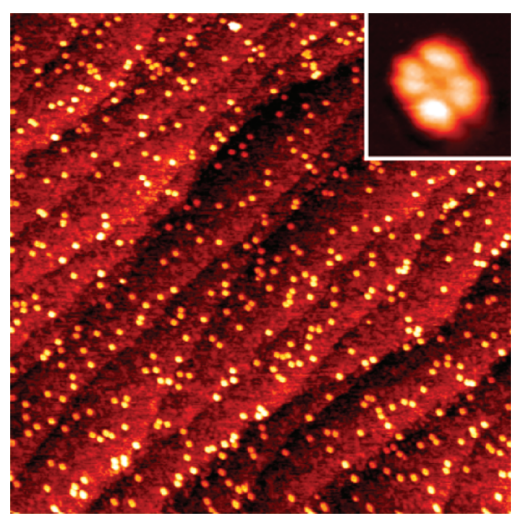

Figure 1. $\mathrm{STM}$ image of dispersed $\mathrm{TbPc}_{2}$ on $\mathrm{Cu}(100)$ acquired prior to the XAS measurements. Inset: Single $\mathrm{TbPc}_{2}$ molecule revealing the fourlobed structure of the top Pc ligand $\left(200 \times 200 \mathrm{~nm}^{2},-0.75 \mathrm{~V}\right.$, $0.18 \mathrm{nA})$. 

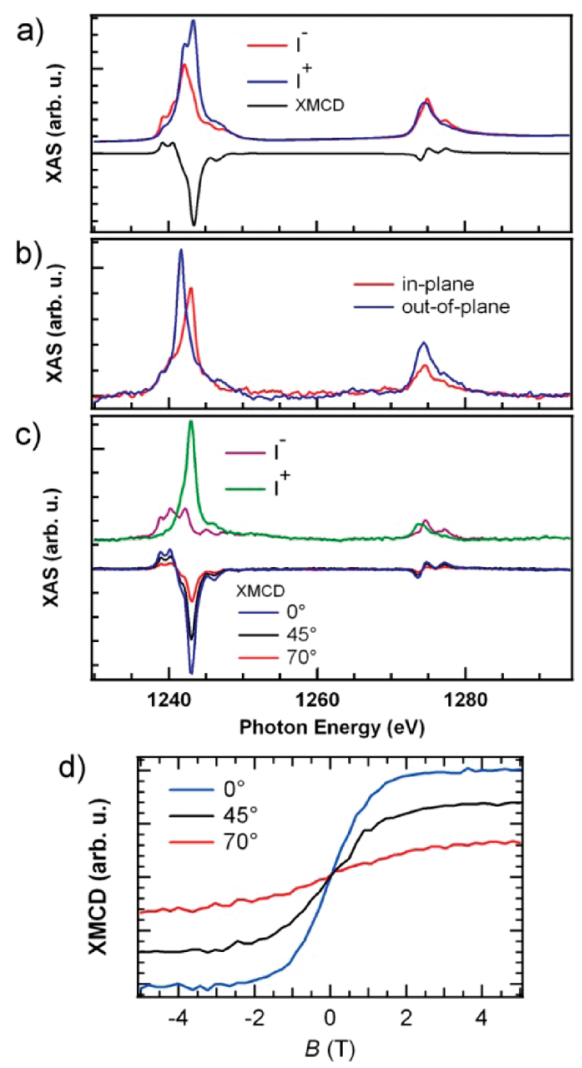

Figure 2. (a) XAS for circular polarized light and corresponding XMCD for neutral $\mathrm{TbPc}_{2}$ powder sample. (b) XAS of $\mathrm{TbPc}_{2}$ on $\mathrm{Cu}(100)$ for linear polarized light parallel to the surface (in-plane) and $20^{\circ}$ to the surface normal (out-of-plane) ( $T=8 \mathrm{~K}, B=50 \mathrm{mT})$. (c) Circular polarized XAS of $\mathrm{TbPc}_{2}$ on $\mathrm{Cu}(100)$ at $0^{\circ}$ (normal) incidence $\left(I^{+}, I^{-}\right)$and $\mathrm{XMCD}$ at $0^{\circ}, 45^{\circ}$, and $70^{\circ}$ incidence ( $T=8 \mathrm{~K}, B=5 \mathrm{~T}$ ). (d) Magnetization curves obtained by the $\mathrm{Tb} M_{5} \mathrm{XMCD}$ intensity for $\mathrm{TbPc}_{2}$ on $\mathrm{Cu}(100)$ at $T=8 \mathrm{~K}$.

XAS spectra for right and left circular polarization $\left(I^{+}\right.$and $I^{-}$, respectively) obtained at the $\mathrm{Tb} M_{4,5}$-edge for the powder sample and adsorbed molecules (background subtracted) are presented in Figure $2 \mathrm{a}$ and $\mathrm{c}$, respectively. We find that the XAS line shape agrees very well with that calculated for a $\mathrm{Tb} 4 f^{8}$ configuration with a $J=6$ ground state. ${ }^{8}$ This is in agreement with the ground state inferred from bulk DC susceptibility and NMR measurements reported in ref 4. Furthermore, the sizable XMCD $\left(I^{-}-I^{+}\right)$intensity $(T=8 \mathrm{~K}, B=5 \mathrm{~T})$ and $M_{4,5} \mathrm{XMCD}$ branching ratio imply the presence of a large orbital moment at the $\mathrm{Tb}$ ion. Figure $2 \mathrm{~b}$ shows the XAS spectra of $\mathrm{TbPc}_{2}$ on $\mathrm{Cu}(100)$ for linear light polarization $(T=8 \mathrm{~K}, B=50 \mathrm{mT})$. The strong linear dichroism signifies that the molecules are oriented and that the $4 f$ shell is subject to a robust crystal-field. The XMCD, shown at incidence angles $\theta=0^{\circ}, 45^{\circ}$, and $70^{\circ}$ after normalization to the integrated XAS intensity (Figure $2 \mathrm{c}$ and d), is strongly anisotropic. While the XAS line shape varies with $\theta$, the XMCD line shape does not change apart from a scaling factor, contrary to what is observed in planar molecular coordination networks. ${ }^{1}$ This finding suggests that the magnetic moment of the $\mathrm{Tb}$ ion follows an Ising-like behavior; i.e., it aligns either parallel or antiparallel to the surface normal, depending on the sign of the applied field, and the angle-dependent $\mathrm{XMCD}$ scales as $\cos \theta$, i.e., as the projection of a perpendicular moment onto the X-ray direction. Such a scaling is also found for the high field values of the magnetization reported in Figure 2d.

The XMCD line shapes of the surface-supported and powder $\mathrm{TbPc}_{2}$ samples are indistinguishable (Figure S1). Despite the very strong anisotropy, neither sample shows magnetic hysteresis down to $8 \mathrm{~K}$, below the blocking temperature of $25-40 \mathrm{~K}$ reported in
Table 1. XMCD Sum Rule and Calculated Magnetic Moments along the Applied Magnetic Field Direction $z$ at $T=8 \mathrm{~K}, B=5 \mathrm{~T}$

\begin{tabular}{lccccccc}
\hline & \multicolumn{2}{c}{ sum rules } & & \multicolumn{4}{c}{ multiplet calculation } \\
\cline { 2 - 3 } \cline { 5 - 7 } angle & $L_{z}$ & $S_{\text {eff }}$ & & $L_{z}$ & $2 S_{z}$ & $T_{z}$ & $S_{\text {eff }}$ \\
\hline $0^{\circ}$ & 3.14 & 4.15 & & 3.05 & 5.89 & -0.24 & 4.43 \\
$45^{\circ}$ & 2.14 & 2.89 & & 2.15 & 4.16 & -0.17 & 3.13 \\
$70^{\circ}$ & 0.97 & 1.42 & & 0.92 & 1.79 & -0.07 & 1.36 \\
$90^{\circ}$ & - & - & & 0.03 & 0.07 & 0 & 0.07 \\
powder & 1.16 & 1.49 & 0.93 & 1.80 & -0.07 & 1.36 \\
\hline
\end{tabular}

ref 4 on the basis of AC susceptibility measurements. This is not surprising given that the time $(\sim 20 \mathrm{~min})$ required to measure a magnetization curve by XMCD is 4 to 7 orders of magnitude larger compared to AC measurements and that the susceptibility is still appreciable below $10 \mathrm{~K}^{4}$

The orbital $\left(L_{z}\right)$ and effective spin $\left(S_{\text {eff }}=2 S_{z}+6 T_{z}\right)$ magnetic moments projected onto the photon incidence direction $z$ are obtained by employing the XMCD sum rules. ${ }^{9} S_{\text {eff }}$ includes the contribution from the anisotropic spin density, i.e., the spin dipole moment $T_{z}$. The isotropic XAS is approximated by $3 / 2\left(I^{+}+I^{-}\right)$, and the number of holes in the $f$-shell is taken as 6 . The results are summarized in Table 1. In addition, the moments were calculated by means of ligand field multiplet theory ${ }^{8}$ using the crystal field parameters reported by Ishikawa et al. ${ }^{4}$ The powder was simulated by calculating the angular average of the moments. The multiplet calculations allow for the direct evaluation of the spin $S_{z}$ and spin dipolar $T_{z}$ moments. The agreement between experiment and calculations is excellent. We conclude that although the interaction strength of the lower Pc ligand with the substrate is sufficient to impede diffusion of the molecules, their ligand field and hence magnetic properties remain unaltered. The $\mathrm{Tb}$ ion presents the full orbital and spin magnetic moment expected for a $J=$ 6 ground state along the easy axis and vanishing magnetization in the hard Pc plane.

Acknowledgment. We thank the ID08 beamline staff at ESRF for technical assistance and S. Brink for help with the synthesis. The work was supported by the ESF (SANMAG \& FunSMARTs II). P.G. acknowledges support from the ERC (StG 203239).

Supporting Information Available: Comparison and angular dependence of Tb XMCD. Tb XAS simulation. Description of synthesis and calculations. Complete ref 1 . This material is available free of charge via the Internet at http://pubs.acs.org.

\section{References}

(1) Gambardella, P.; et al. Nat. Mater. 2009, 8, 189.

(2) Mannini, M.; Sainctavit, P.; Sessoli, R.; dit Moulin, C. C.; Pineider, F.; Arrio, M.-A.; Cornia, A.; Gatteschi, D. Chem.-Eur. J. 2008, 14, 7530.

(3) Mannini, M.; Pineider, F.; Sainctavit, P.; Danieli, C.; Otero, E.; Sciancalopore, C.; Talarico, A. M.; Arrio, M.-A.; Cornia, A.; Gatteschi, D.; Sessoli, R. Nat. Mater. 2009, 8, 194

(4) (a) Ishikawa, N.; Sugita, M.; Ishikawa, T.; Koshihara, S.; Kaizu, Y. J. Am. Chem. Soc. 2003, 125, 8694. (b) Ishikawa, N.; Sugita, M.; Okubo, T.; Tanaka, N.; Iino, T.; Kaizu, Y. Inorg. Chem. 2003, 42, 2440.

(5) Vitali, L.; Fabris, S.; Mosca Conte, A.; Brink, S.; Ruben, M.; Baroni, S.; Kern, K. Nano Lett. 2008, 8, 3364.

(6) (a) Katoh, K.; Yoshida, Y.; Yamashita, M.; Miyasaka, H.; Breedlove, B. K.; Kajiwara, T.; Takaishi, S.; Ishikawa, N.; Isshiki, H.; Zhang, Y. F.; Komeda, T.; Yamagishi, M.; Takeya, J. J. Am. Chem. Soc. 2009, 131, 9967. (b) Zhang, Y. F.; Ishiki, H.; Katoh, K.; Yoshida, Y.; Yamashita, M.; Miyasaka, H.; Breedlove, B. K.; Kajiwara, T.; Takaishi, S.; Komeda, T. J. Phys. Chem. C 2009, 113, 9826.

(7) Branzoli, F.; Carretta, P.; Filibian, M.; Zoppellaro, G.; Graf, M. J.; GalanMascaros, J. R.; Fuhr, O.; Brink, S.; Ruben, M. J. Am. Chem. Soc. 2009, $131,4387$.

(8) Thole, B. T.; van der Laan, G.; Fuggle, J. C.; Sawatzky, G. A.; Karnatak, R. C.; Esteva, J.-M. Phys. Rev. B 1985, 32, 5107.

(9) (a) Thole, B. T.; Carra, P.; Sette, F.; van der Laan, G. Phys. Rev. Lett. 1992, 68, 1943. (b) Carra, P.; Thole, B. T.; Altarelli, M.; Wang, X. Phys. Rev. Lett. 1993, 70, 694.

JA105124R 